\title{
Comparing static Gantt and mosaic charts for visualization of task schedules
}

\author{
Saturnino Luz \\ School of Computer Science and Statistics \\ Trinity College \\ Dublin 2, Ireland \\ Email:luzs@cs.tcd.ie
}

\author{
Masood Masoodian \\ Department of Computer Science \\ The University of Waikato \\ Hamilton, New Zealand \\ Email:m.masoodian@cs.waikato.ac.nz
}

\begin{abstract}
A mosaic chart has been proposed for representation of events on a timeline. While early studies demonstrated the effectiveness of mosaics in supporting visualization of multimedia records on a meeting browser, the usability of mosaics as a static timeline visualization has not been studied in more general settings. This paper investigates the use of the mosaic charts for visualization of project schedules. A user study was conducted to compare a building project schedule encoded alternatively as a mosaic or as a Gantt chart. Although the study focused on static graphs, for which the Gantt technique is usually very effective, results showed that the users were as fast and accurate at answering the questions using the mosaic representation as they were using Gantt charts. The analysis and experiment indicated algorithmic, space-filling and interpretation limitations of the mosaic technique. We suggest possible design improvements to overcome some of these limitations.
\end{abstract}

Keywords-Timelines; Gantt charts; Temporal mosaic, Screen design; Information Visualization;

\section{INTRODUCTION}

Visualization of multiple chronologies spanning a common time period is usually done through timelines. A ubiquitous example of timeline is the Gantt chart, a tool which has been used for representation of task schedules since the beginning of the last century [1]. The fact that Gantt charts are still widely used in a number of areas testifies to how intuitive and effective the idea of representing temporal data streams as rectangular bars stretching along a timeline can be.

Although Gantt charts were initially conceived for the paper medium, where space constraints are not usually a cause for concern, the representational recipe for Gantt charts has undergone little change since Henry Gantt's time. In fact, a case could be made that because Gantt charts serve broader group coordination purposes as shared organizational artifacts they are irrevocably bound to the paper medium. Tufte [2], for instance, acknowledges the strengths of timeline visualizations, but elsewhere he claims that Gantt charts are ill-suited to computer screens and can only be useful if drawn on large sheets of paper and made available in public spaces [3]. Despite these concerns the static metaphor of Gantt charts also carries through to interactive contexts, and timelines have evolved and mi- grated into the electronic medium [4]. They are now widely used in a variety of systems that deal with sequential data, including project management applications, browsing of multimedia collections [5] and meetings [6], representation of patient history and planning in health informatics [7], [8], visualization of travel itinerary information across timezones [9], [10] and many others.

As timelines were incorporated into interactive systems, certain innovations were introduced which facilitated data input and presentation of multiple views. However, the basic design concept remained roughly the same. While the coordination roles of timeline artifacts such as paper Gantt charts have been either neglected or assumed to be performed elsewhere (through, for instance, integrated collaborative environments, etc) the screen real-estate limitations of the new medium have often been addressed through standard graphical user interface techniques such as scroll-bars, zoom and pane, and overview plus detail.

Leaving aside the coordination issue, which this paper will not address, and focusing solely on the timeline as a user interface component, one could argue that the transition to the electronic medium facilitated by the aforementioned techniques has not been entirely satisfactory either. For instance, if vertical scroll-bars are used the user's ability to align events decreases. Even in cases where the entire diagram fits on the same screen one needs to consider that visual sensitivity to spatial alignment decreases as the distance between the targets increases [11]. The difficulty is also accentuated if alignment needs to be recognized across intervening parallel lines, as is often the case in Gantt charts. The basic design premise of Gantt charts, i.e. that each event type be allocated a horizontal line, contributes to making these limitations quite common in practice. As the number of event types (task types, in Gantt charts) increases, so does the height of the chart. This results in wasteful space allocation since most diagrams will be dominated by blank spaces representing inactivity. As the graph grows vertically with the number of event types, the user's ability to recognize concurrent events and exclusively inactive intervals is also greatly impaired.

Luz \& Masoodian [12] introduced "temporal mosaics" as an alternative to timelines for visualization of multiple 


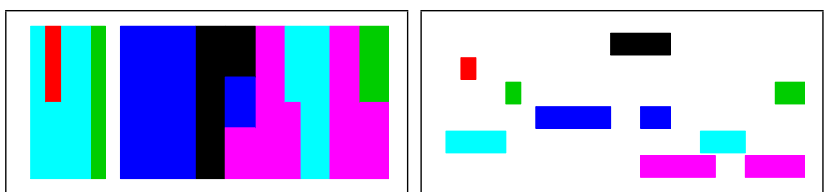

Figure 1. The same set of events represented in temporal mosaic (left) and Gantt (right).

streams of events and their dependencies and relationships. Unlike a timeline, which grows vertically on demand, a temporal mosaic is a form of visualization that starts with a fixed height and allocates space proportionally to the number of overlapping tasks in a given time interval. Task types are no longer tied to particular lines but are represented through colour codes, as illustrated in Figure 1. Temporal mosaics preserve the representation of overview, even as the available drawing area is reduced, and facilitate the detection of concurrent and overlapping events, even as the number of events to be represented increases.

Temporal mosaics have been used in a mobile meeting browsing system [13] and evaluated for usability and effectiveness in a multimedia browsing task [12]. Although the evaluation showed that users performed better when using the mosaic-based browser, the tasks they were asked to perform were not typical schedule visualization tasks, and involved dynamic, interactive elements such as playing audio events and alternating between timeline and textual views.

This paper addresses these issues by assessing the use of mosaics in a static (non-interactive) and more familiar schedule visualization task. A user study is reported which compared user performance at answering questions about building schedules represented in mosaic and Gantt charts. Results are presented for accuracy, time to answer, and difficulty ratings. The main findings are discussed, limitations of the mosaic technique are analysed, and methods and guidelines for addressing those limitations are proposed.

\section{Evaluation}

As mentioned above, the mosaic technique has been found in a previous study [12] to be effective for visualization of meeting recordings, where the number of event types is relatively small, and the vertical axis can be faceted to group events according to media source (audio, text). In contrast, the evaluation described in this section compares the mosaic visualization with the more familiar Gantt visualization, in a more complex context where the number of event types is much greater than the number of event types used in the previous study.

Furthermore, although we anticipated that temporal mosaics would be most useful for interactive dynamic visualizations systems, particularly for small-screen devices, in this comparison we were mostly interested in finding out whether users could readily understand the mosaic mapping in a static representation, and perform temporal inferences based on it at least as effectively as when using the Gantt chart. Therefore, we chose a building schedule visualization task as the basis of our evaluation.

\section{A. Method}

In this study, a single project schedule was displayed to the participants as mosaic or Gantt timelines in conjunction with a question about the schedule, followed by a difficulty rating question. The project schedule used in the experiment is shown in Figure 2, in its mosaic and timeline forms.

A within-subject design was employed. All subjects were given a written tutorial explaining both forms of the visualization. The tutorial consisted of a short text which described the types of visualization to be used (i.e. Gantt and mosaic), illustrating each type with graphs similar to those actually used in the study. At the end of this tutorial the subjects were directed to the live visualization trial.

Table I

SAMPLE QUESTIONS.

\begin{tabular}{cl}
\hline Type & sample question \\
\hline $\mathrm{e}$ & No more than 3 tasks should be scheduled for the same day. \\
& Is this a problem with the current plan? \\
$\mathrm{i} \quad$ How many days are free in the first two weeks? \\
$\mathrm{c} \quad$ All the windows are installed by the same person and should \\
\\
happen in the same days. Is this possible in the current \\
schedule? \\
$\mathrm{d} \quad$ How many days does the bathroom plumbing take in total? \\
$\mathrm{o} \quad$ Painting a room should finish before its carpet can be installed. \\
\\
Is this a problem for the bedroom schedule?
\end{tabular}

Each trial consisted of a set of 10 pairs of questions, displayed one at a time in conjunction with either the mosaic or Gantt representation of the project chart. For each pair, we randomly allocated images to questions according to a uniform probability distribution. Therefore, in addition to randomizing the presentation sequence so as to minimize order effects, we randomized the question-image pairs in order to minimize possible learning effects. The types of questions employed in this trial were exclusion (e), duration (d), inactivity (i), concurrence (c) and ordering (o). Examples of each type of questions are shown in Table I.

All questions were multiple choice and the choices were presented through drop-down lists. Once the participants selected their response from a list of choices, the system recorded the time taken to answer the question, and then presented them with a request to rate the difficulty of the question they had just answered on a Likert scale ranging from 1 (easy) to 7 (difficult). The participants were told that the time taken to rate a question would not count towards the total task completion time. A prize (book voucher) was offered to the participant that answered the questions most accurately in the shortest time.

The study had 23 participants: 14 were male, 9 female; 11 were academics, 7 were students and 5 had other occupations. The system recorded 460 answers and ratings. 


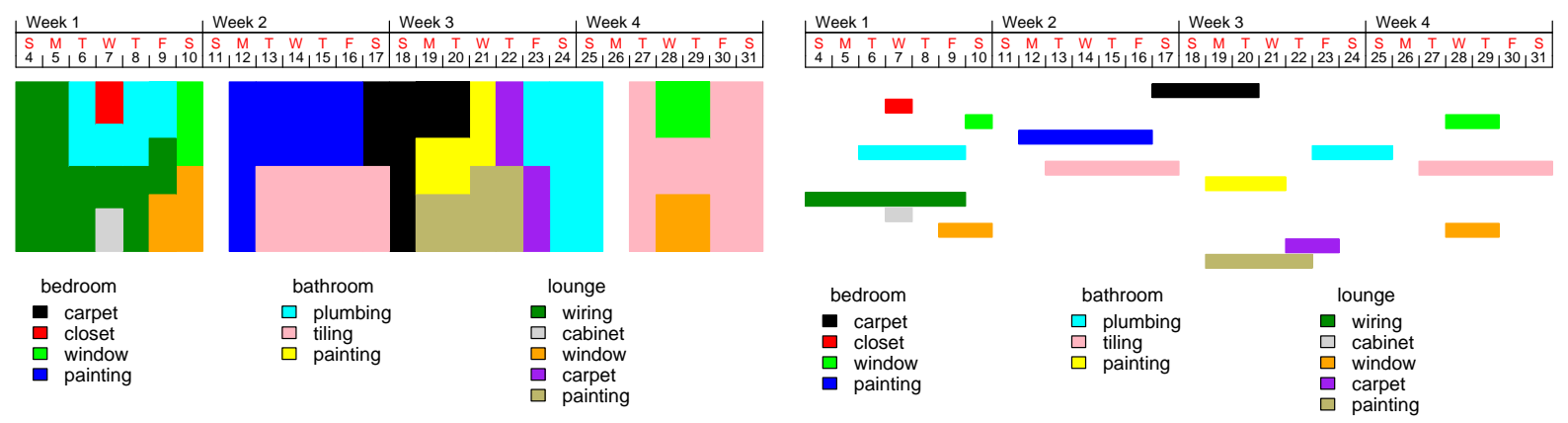

Figure 2. Scheduling constraints used in the study, represented as mosaic and Gantt charts.

\section{B. Results}

Remarkably, very little difference was observed in accuracy and answer time results for Gantt and mosaic charts. Mean error rates were practically identical. Macro-averaged (over mean error per question) the error rates were $15 \%$ for mosaic and $15.1 \%$ for the Gantt chart. Mosaic users took an average of 20.4 seconds to answer a question while Gantt timeline users took 21.2 seconds, but the difference was not found to be statistically significant $(\mathrm{t}[436]=0.5, \mathrm{p}=0.5)$.

Difficulty ratings were also very similar, with mosaic being rated slightly less difficult than Gantt timelines on average (2.3 versus 2.4 , not significant). Since participants were randomly presented with both alternative visualizations for each question type, it is likely that these ratings accurately reflect their assessment of the relative difficulties of mosaic and timeline. Time-rating correlations were found to be quite robust. Pearson's product-moment correlation was $0.68(p<0.001)$ for Gantt and $0.62(p<0.004)$ for mosaic, showing that subjects tended to find more difficult those questions they took longer to answer.

A detailed breakdown of answer times and error rates per question is shown in Figure 3. While some questions were probably too easy, as is the case of the ones about inactivity (11 and 12), other seemingly straightforward questions such as question 4 proved surprisingly challenging. Question 4 reads: "All the windows are installed by the same person, and should be installed in the same days. Is this possible in the current schedule?", requiring a simple concurrence detection task. It is possible that the problems found by the subjects in answering this question stem from difficulties in interpreting the text itself, rather than the graphics. However, the low accuracy observed in both conditions seems to indicate otherwise.

We also grouped the answers according to type (concurrence, inactivity, duration, exclusion and ordering) and plotted the combined results for speed, accuracy and ratings (Figure 4). The only group for which one of the visualization styles had a noticeable advantage was the group of concurrence assessment questions, for which mosaic users were faster (and practically as accurate as timeline users).

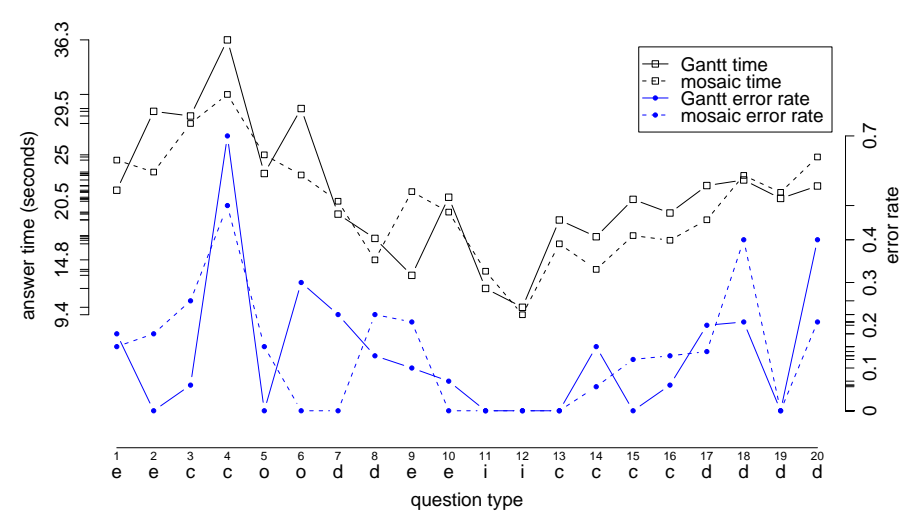

Figure 3. Answer times and error rates.

\section{Discussion}

Temporal mosaic matched the Gantt timeline visualization very closely for all types of event relationship detection tasks. Mosaic users performed well at detecting event overlaps, as expected, but they also did well at assessing event duration. The latter is somewhat surprising, since in the meeting visualization task evaluation carried out previously [12] users often mistakenly assumed event duration to be proportional to the area occupied by the event representation on the graph. We infer from this that since in the schedule visualization study participants were presented with both Gantt and mosaic charts, the former (for which interpretation of duration is clearly unambiguous) helped them form the correct mental model when using the latter. The mosaic visualization should, therefore, aim to utilize this mental model of timeline that most people already have due to their familiarity with Gantt charts. An improvement to the mosaic design along these lines could be to draw thin horizontal bars spanning the maximum width of each mosaic area as a way of suggesting the right mapping of width to time duration.

As noted above, of the questions that required detection of concurrency, question number 4 can be regarded as "difficult", specially if answered against a Gantt chart. They took a long time to answer it but still made many mistakes. 

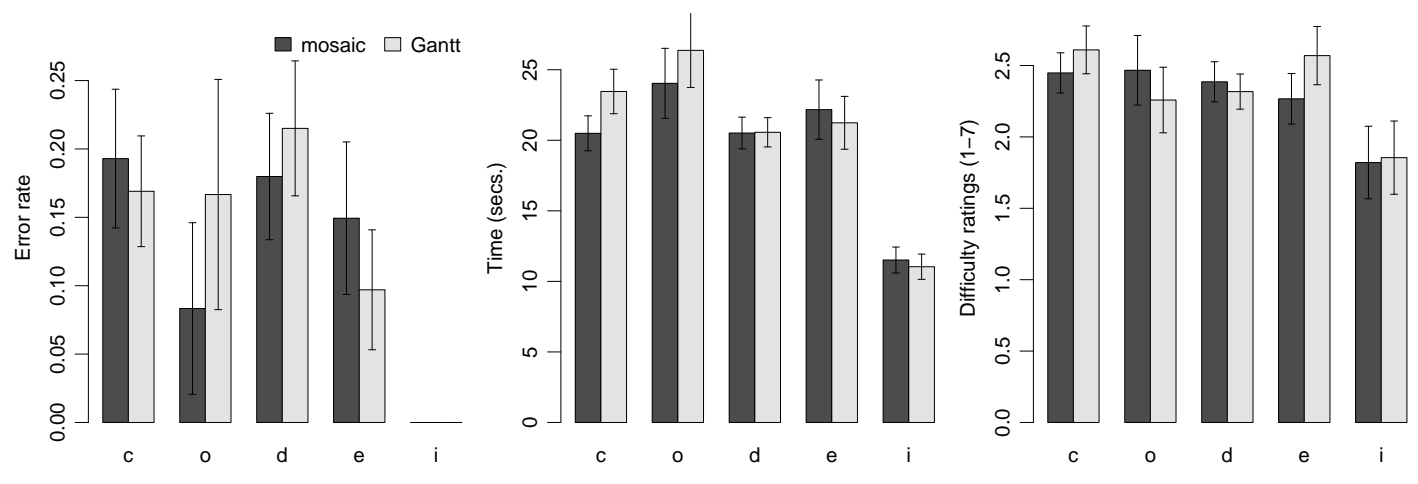

Figure 4. Answer time, accuracy and ratings by type of question.

Similarly, the questions concerning inactivity (11 and 12) can be regarded as "easy" in both visualizations. Question 18 illustrates the issue of misinterpretation of event duration in the mosaic visualization discussed above. Mosaic users did not take too long to answer the question but eventually answered it incorrectly. Closer examination of question 18, which reads "How many days does the bathroom tiling take in total?", also suggests that the respondents might have been misled by the placement of the legend for bathroom directly under week two, which happens to be exactly where the first period of tiling activity ends (see Figure 2). This was particularly problematic for the mosaic representation since it happened to extend right down to the beginning of the legend, causing its users to overlook the second period of tiling activity which starts much later, in week four.

Finally, the study revealed a shortcoming of the mosaic drawing algorithm, which sometimes produces the undesirable result of rendering an event that should be drawn as a single contiguous rectangle as two rectangles discontinuous ("broken") across lines. An example of such discontinuous drawings occurred for the lounge carpeting task (days 22 and 23). Figure 5 illustrates the problem: the two rectangles marked (a) in the zoom area should have been drawn as a single rectangle. This seemingly caused the high number of errors made by mosaic users in question 2 ("Putting carpet in a room and painting that room can't coincide. Is this a problem for the lounge schedule?") while Gantt users always answered correctly. A few of the participants pointed out that they were confused by the fact that the area corresponding to carpeting appeared not as a single rectangle spanning the $22 \mathrm{nd}$ and $23 \mathrm{rd}$ but as two adjacent rectangles, one at the top and one at the bottom of the screen. This indicates that discontinuity is undesirable. The question however is, can discontinuity always be avoided in mosaics? In the next section we address this question.

\section{Discontinuity IN MOSAICS}

Let us start by formalizing the issue of discontinuity in mosaic visualization based on Algorithm 1, which is a

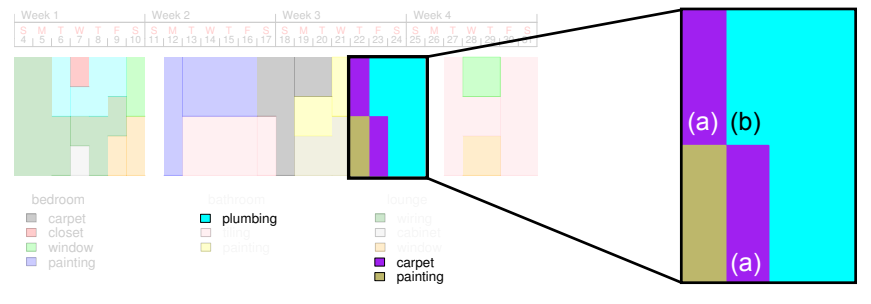

Figure 5. Example of discontinuity.

simplified version of the original mosaic drawing algorithm [12].

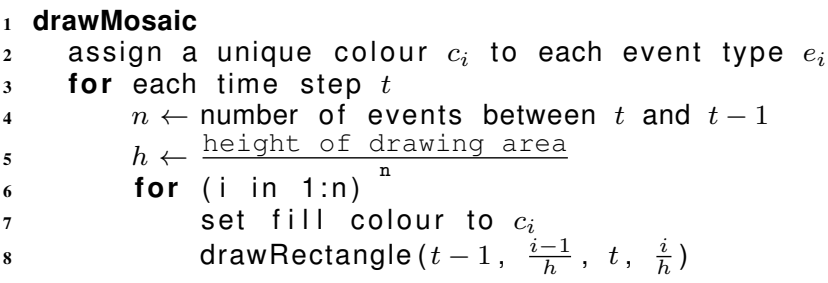

Algorithm 1. Mosaic drawing.

We say that a representation for an event $e$ on the mosaic is a discontinuous representation if $e$ extends from time $t_{b}$ to $t_{e}$ and there are times $t_{i}$ and $t_{j}=t_{i}+1\left(t_{b} \leq t_{i}<t_{e}\right)$ such that the vertical sides of the rectangles representing $e$ at $t_{i}$ and $t_{j}$ do not coincide or overlap. In the case of Figure 5 the problem could have been avoided by reordering the plumbing event (b) to be drawn below the carpeting event (a). However, this forward ordering strategy will not always guarantee continuity. In fact, for any 2 -interval sequence consisting of an interval $t$ with $m>3$ events, immediately followed by an interval $t^{\prime}$, containing $m-2$ events that also occur in $t$, Algorithm 1 will produce at least one discontinuous representation. This is also true of the algorithm proposed in [12].

Let $e_{1}, \ldots, e_{m}$ be the $m$ events represented in interval $t$. Assume that $t$ is followed by interval $t^{\prime}$ containing $e_{1}^{\prime}, \ldots, e_{m-2}^{\prime}$. If $h$ is the height of each rectangle for each 
$e_{i}$ in $t$, then the height $h^{\prime}$ of each $e_{i}^{\prime}$ will be:

$$
\begin{aligned}
h^{\prime}(m-2) & =h m \\
h^{\prime} & =h \frac{m}{m-2}
\end{aligned}
$$

At best all $m$ events in $t$ will be ordered, say, from $e_{1}$ to $e_{m}$, and all events in $t^{\prime}$ correspondingly ordered, i.e. $\left(e_{1}, \ldots, e_{m-2}\right)$ so that, for all $e_{i}$ in $t$ and $e_{j}^{\prime}$ in $t^{\prime}, i=j$. Let $h_{0}\left(e_{i}\right)$ be the vertical coordinate for the top side of the rectangle that represents $e_{i}$, and $h_{1}\left(e_{i}\right)$ the coordinate for the rectangle's bottom corner. Clearly, in order for continuity to hold, $\forall_{i} h_{1}\left(e_{i}\right)>h_{0}\left(e_{i}^{\prime}\right)$. According to (1), the general pattern of the sequence is the following:

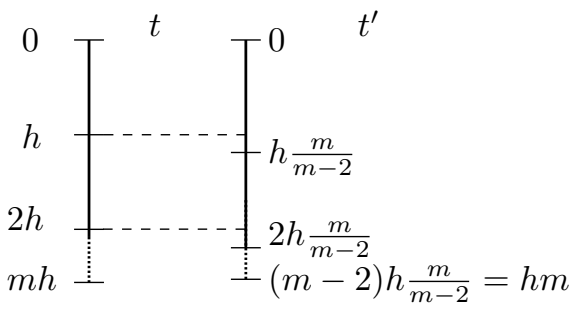

Therefore, for $h_{1}\left(e_{i}\right)>h_{0}\left(e_{i}^{\prime}\right)$ to hold for all $i$ we need:

$$
\begin{aligned}
i h & >\frac{(i-1) h m}{m-2} \\
i & <\frac{m}{2}
\end{aligned}
$$

In other words, for $i=m-2$ inequation (2) can only be satisfied by $m<4$.

\section{A. Strategies for avoiding discontinuity}

A possible strategy for dealing with the discontinuity issue would be to alter the mosaic drawing algorithm so as to allow the rectangles (tiles) to have variable heights within intervals. In this case, vertical space would no longer be allocated equally among concurrent events. Before drawing a rectangle for event $e^{\prime}$ we would check if drawing the next rectangle in the same time interval would result in discontinuity. If so, we would reduce the height $h_{1}\left(e^{\prime}\right)$ so as to keep $h_{0}\left(e_{i+1}^{\prime}\right)<h_{1}\left(e_{i+1}\right)$. The visual result would be a mosaic in which the events at the top of the graph would seem "compressed" in certain intervals, producing a visually less intuitive design.

Another possibility would be to allow all segments (not only the next segment to be drawn) to be reordered once the continuity constrain has been violated. However, this strategy would be much costlier, since an exponentially large space of possible arrangements would need to be searched. Moreover, it does not guarantee continuity, as can be easily verified by trying to lay out a set of events $\left\{e_{1}, \ldots, e_{6}\right\}$ on a 3 -interval mosaic, such that the first interval contains $\left\{e_{3}, \ldots, e_{6}\right\}$, the second contains $\left\{e_{1}, \ldots, e_{6}\right\}$ and the third $\left\{e_{1}, \ldots, e_{4}\right\}$. Fortunately, such unsolvable continuity constraints rarely arise in practice. Therefore, a heuristic that seeks to minimize the number of discontinuous representations by looking ahead one interval and reordering streams appropriately before drawing will suffice in most situations. The required modification to the drawing algorithm of temporal mosaic is straightforward. Algorithm 1 can be modified so that the events occurring between $t-2$ and $t-1$ are stored in a vector, and the events between $t-1$ and $t$ are reordered prior to drawing so that the events that also occurred in the previous interval are drawn as closely to their height in that interval as possible.

\section{DESIGN PRINCIPLES FOR DRAWING MOSAICS}

The results of the study presented in the this paper can be used to define a series of visual design principles which can be incorporated into the algorithm for drawing temporal mosaics to improve its effectiveness.

In terms of visual variables [14], the basic difference between the temporal mosaic and a conventional timeline is that the latter relies on position, size (and occasionally colour) as its core attributes while the former trades position for colour (and optionally texture) as the plot's underlying visual variables. An event representation based on colour rather than position risks undermining visual continuity. Preserving visual continuity is the most important aspect of conventional timelines such as the Gantt chart, while space efficiency is the most beneficial aspect of temporal mosaics. Therefore, the overall aim of the design principles outlined below is to preserve, as much as possible, the visual continuity of individual tasks across the time axis, while also utilizing space more efficiently across the tasks axis.

\section{A. Avoiding discontinuity}

Visual discontinuity is the least desirable aspect of the mosaics visualizations, and therefore should be avoided when possible. As demonstrated, even though there are some cases where discontinuity cannot be avoided, an approximation algorithm can be employed which deals with the cases in which it can be avoided, as described above.

\section{B. Visual alignment against the time axis}

Timeline visualizations such as Gantt charts allow viewers to scan space reserved for each task, which is aligned against the time axis, to see all the instances of that particular task across time. Although this is not the case for mosaics, as no specific area is reserved for each task, it may still be possible to provide some visual alignment against the time axis especially for tasks that occur more frequently. One way of achieving this is to analyze the entire tasks dataset to calculate the frequency, or duration, of each task and then order the tasks according to their frequency or duration, so that task mosaics can be drawn in the order of their frequency or duration (e.g. top to bottom along the tasks axis). Although this does not guarantee alignment, it is likely 
to lead to higher frequency tasks being drawn in a narrower visual space along the time axis (e.g. always closer to the top).

\section{Selection of colours}

Selection of colours to represent different tasks in Gantt charts is not usually very important, as space is used to preserve the visual continuity, and separation, of individual tasks. In temporal mosaic, on the other hand, the association between colours and different tasks is crucial to the effectiveness of the visualization. It is very important to carefully choose colours that would allow individual tasks to be easily identified and separated from other tasks, particularly when the number of tasks increases. There are a number of colour selection criterion, based on graphic design principles, that could be adopted. The most important criteria is that tasks that are likely to co-occur, and hence be drawn close to one another, should be complementary so that they are easily distinguished from one another. This selection criteria could be combined with the task ordering and drawing sequence, as described above.

\section{Enhancing visual continuity}

Visual continuity of individual tasks could also be enhanced by combining some elements of Gantt timelines with temporal mosaics. Although such improvements can be added more easily in interactive visualization systems (e.g. by overlaying Gantt timelines over mosaics), in static visualizations connecting lines could for example be used to join occurrences of different instances of each task across the entire mosaic visualization.

\section{CONCLUSION}

The study presented above demonstrated that, despite its different visual style, temporal mosaic preserve the intuitiveness of timelines while using space more effectively. It also showed that first-time users of temporal mosaic are able to match the performance of Gantt users for speed and accuracy. Results and user feedback indidated possible improvements and guidelines for drawing mosaic charts.

Although our focus here has mainly been on evaluation of "static" mosaic and timeline visualizations, we believe that much can be gained by providing interactive alternatives to these static visualizations. Therefore, in parallel to this research, we have also been developing a prototype tool for interactive creation, visualization and manipulation of scheduling tasks using Gantt and mosaic techniques, as well as different combinations of both [15].

\section{ACKNOWLEDGMENT}

Saturnino Luz is supported by the Science Foundation Ireland (Grant 07/CE/I1142) as part of the Centre for Next Generation Localisation (www.cngl.ie) at Trinity College Dublin.

\section{REFERENCES}

[1] H. Gantt, "Organizing for work," Industrial Management, vol. 58, pp. 89-93, 1919.

[2] E. R. Tufte, The Visual Display of Quantitative Information, 2nd ed. Graphics Press, 2001.

[3] — , "Project management graphics: ET notebooks (Ask ET," http://www.edwardtufte.com/bboard/ q-and-a-fetch-msg?msg \_id=000076, 2002, (retrieved November 2010).

[4] S. F. Silva and T. Catarci, "Visualization of linear timeoriented data: A survey," in Procs. of the International Conference on Web Information Systems Engineering (WISE). Hong Kong: IEEE, 2000, pp. 310-319.

[5] D. Huynh, S. Drucker, P. Baudisch, and C. Wong, "Time quilt: scaling up zoomable photo browsers for large, unstructured photo collections," in CHI '05: Human Factors in Computing Systems. ACM, 2005, pp. 1937-1940.

[6] M.-M. Bouamrane and S. Luz, "Meeting browsing," Multimedia Systems, vol. 12, no. 4-5, pp. 439-457, 2007.

[7] S. Cousins and M. Kahn, "The visual display of temporal information," Artif. Intell. in Medicine, vol. 3, no. 6, pp. 341357, 1991.

[8] R. Kosara and S. Miksch, "Visualization methods for data analysis and planning in medical applications," International Journal of Medical Informatics, vol. 68, no. 1-3, pp. 141153, 2002.

[9] M. Apperley, D. Fletcher, B. Rogers, and K. Thomson, "Interactive visualisation of a travel itinerary," in AVI 'OO: Proc. of Advanced Visual Interfaces. ACM, 2000, pp. 221226.

[10] M. Masoodian, D. Budd, and B. Rogers, "A comparison of linear and calendar travel itinerary visualizations for personal digital assistants," in Procs. of OZCHI'04: Conf. on HumanComputer Interaction, 2004, pp. 124-133.

[11] S. J. Waugh and D. M. Levi, "Spatial alignment across gaps: contributions of orientation and spatial scale," Journal of the Optical Society of America, vol. 12, no. 10, pp. 2305-2317, 1995.

[12] S. Luz and M. Masoodian, "Visualisation of parallel data streams with temporal mosaics," in Proc. of the 11th Intl. Conf. on Information Visualisation. Zurich: IEEE Computer Soc., 2007, pp. 196-202.

[13] — , "Visualisation of meeting records on mobile devices," in Handbook of Research on User Interface Design and Evaluation for Mobile Technology, J. Lumsden, Ed. IGI, 2008, pp. 1049-1067.

[14] J. Bertin, Sémiologie graphique. Gauthier-Villars Mouton, 1967.

[15] S. Luz and M. Masoodian, "Improving focus and context awareness in interactive visualization of time lines," in $\mathrm{HCI}$ '10: Proceedings of the 24th BCS Conference on Human Computer Interaction, British Computer Society. Dundee, Scotland: ACM Press, 2010. 